\title{
An Elementary Peircean and Category-Theoretic Reading of Being and Event, Logics of Worlds and The Immanence of Truths
}

Badiou's magnum opus, i.e. his trilogy Being and Event ${ }^{1}$, Logics of Worlds ${ }^{2}$, and The Immanence of Truths ${ }^{3}$, with all its dubious statements, deviations, and controversial formulas, but also with all its courage, finesse, and dedicated hard work, represents the greatest effort by a philosopher in the last thirty years to extensively confront the power of contemporary mathematical invention (e.g. forcing, toposes, and large cardinals). The enterprise has not passed unnoticed, but has mainly attracted the attention of some strong detractors, as well as a small community of worshippers. Both extremes are unjust as regards this amazing work that now spans three decades and which should be hailed as a unique contribution to knowledge, and we will try here to present a simple, hopefully balanced, view of the trilogy. In our reading, we will use the force of Peirce's logical architecture and of some high conceptual instances of Category Theory. In Section 1, we explore some basic methodological connections, from a very intuitive dialectical perspective between the three shutters of the trilogy. In Section 2, the dialectics are specified through Peirce's three phenomenological categories and some adjunctions in Category Theory. Finally, in Section 3, we present some open problems related to our simplified, elementary understanding of Badiou's work.

\section{Some dialectical forces in the trilogy}

The main polarities in the foundations of mathematics in the $20^{\text {th }}$ century are Set Theory and Category Theory. On one hand, Set Theory proposes to understand a mathematical object (a "set") through its analytical decomposition: looking at the interior, the elements comprise a set. Some of the axioms of Set Theory are not simple, since they pretend to capture the structural evolution of

1 Alain Badiou, L'Être et l'événement, Seuil, Paris 1988, translated into English by Oliver Feltham: Being and Event, Continuum, London 2008.

2 Alain Badiou, Logiques des mondes, Seuil, Paris 2006, translated into English by Alberto Toscano: Logics of Worlds, Continuum, London 2009.

3 Alain Badiou, L'Immanence des vérités, Fayard, Paris 2018. 
infinities (e.g. the Replacement Axiom, the Foundation Axiom). The size of the continuum (represented in Set Theory as the set of real numbers) even goes beyond the usual axioms, as Cohen showed in 1963 with the invention of forcing. Being and Event explores the uses of Set Theory and forcing to propose a static ontology of mathematics, to be extended to general thought. On the other hand, Category Theory proposes to understand a mathematical object (e.g. "identity", "representable functor") through its synthetic composition: looking at the exterior, the ambient relations ("aura") comprise an identity. The axioms of Category Theory are trivial, but extremely powerful: from practically nothing, the theory captures the mathematical behaviour of apparently distant regions (logic, topology, algebra, arithmetic, differential geometry, etc.). Going further, Grothendieck's Topos Theory (1962) axiomatises many features of set universes inter-spread with geometrical objects. Logics of Worlds explores the applications of Category Theory and toposes to offer a dynamic ontology of mathematics, to be extended to general thought.

The singular of the first monograph contrasts with the plural of the second one: the Absolute searched for in Being and Event is contrasted with the Relative sought in Logics of Worlds. Going beyond the Relative, The Immanence of Truths comes back to the One, and deciphers some approximations of an Absolute Universal, through the many layers of large cardinals. Badiou's commitment is again unique: going painstakingly through the very technical contributions of Cantor, Cohen, Gödel, Jech, Jensen, Kanamori, Kunen, Mostowski, Ramsey, Scott, and Woodin, the many contrasting features of large cardinals are explained to the layman, often with a penetrating poetic language, and are related to politics, culture, and essentially to humanity at large. To be complete (but we will not, of course, require such a commitment from Badiou, who has already done more than enough for generations to come!), a fourth shutter (some sort of "Universels relatifs") of the tetralogy should study the universals inscribed in Topos Theory, i.e. classifier toposes and geometric logics. ${ }^{4}$ In this way, two singulars (L'Etre, L'Immanence) and two plurals (Logiques, Universels) would produce a beautiful counterpoint weaving of the contemporary mathematical foundations. This "fourth shutter" could also be extended to the promising new perspectives offered by Homotopy

4 See, for example, Olivia Caramello, Theories, Sites, Toposes, Oxford University Press, Oxford 2018. 
Type Theory 5 , where some strong connections between geometry and computation reveal a structural universality of which Badiou may be very fond.

Three fundamental ideas provide the backbone of Cohen's forcing: (1C) at the base, the use of a countable standard model of the Zermelo-Fraenkel axioms for Set Theory; (2C) at the approximation level, the use of an order on which forcing conditions are inscribed; $(3 \mathrm{C})$ at the ideal level (or point at infinity), the construction of a generic filter that offers some completeness conditions to provide independence proofs. Many passages of Being and Event are related to situations $(1 \mathrm{C})-(3 \mathrm{C})^{6}$ :

$(1 \mathrm{C})$

"[...] the demonstration that every truth is necessarily infinite" (p. 328).

"A truth (if it exists) must be an infinite part of the situation, because for every finite part one can always say that it has already been discerned and classified by knowledge" (p. 333).

"we shall install ourselves in a multiple which is fixed once and for all, a multiple which is very rich in properties (it 'reflects' a significant part of general ontology) yet very poor in quantity (it is denumerable)" (p. 356).

"This multiple will be both the basic material for the construction of the indiscernible (whose elements will be extracted from it), and the place of its intelligibility" (p. 357).

$(2 \mathrm{C})$

"the study of local or finite forms of a procedure of fidelity" (p. 327).

"the minimal gesture of fidelity: the observation of a connection (or non-connection)” (p. 329).

"A faithful procedure has as its infinite horizon being-in-truth" (p. 339).

"Using a transparent algebra, we will note $x(+)$ the fact that the multiple $x$ is recognized as being connected to the name of the event, and $x(-)$ that it is recognized as non-connected. [...] We will term enquiry any finite set of such minimal reports. [...] It is the enquiry which lies behind the resemblance of the procedure of fidelity to a knowledge" (pp. 330-331).

Homotopy Type Theory, Univalent Foundations Project, Princeton 2018.

6 The citation numbers refer to pages in the English translation: Alain Badiou, Being and Event. Emphases in bold in the quotes are mine. 
"The concept of order is central here, because it permits us to distinguish multiples which are 'richer' in sense than others; even if, in terms of belonging, they are all elements of the supposed indiscernible" (p. 362).

"a condition is useless [...] if it does not tolerate any aleatory progress in the conditioning” (p. 364).

"Order, compatibility and choice must, in all cases, structure every set of conditions” (p. 364).

"We find ourselves here at the threshold of a decisive advance, in which the concept of the 'generic' [...] will be defined and articulated in such a manner that it will found the very being of any truth.” (p. 327)

"We shall therefore say: a truth is the infinite positive total - the gathering together of $x(+)^{\text {'s }}$ - of a procedure of fidelity which, for each and every determinant of the encyclopaedia, contains at least one enquiry which avoids it.

Such a procedure will be said to be generic" (p. 338).

“The generic is the being-multiple of a truth" (p. 338).

"Thus, any veracity in the extension will allow itself to be conditioned in the situation. [...] an inhabitant is in the position of a subject of truth: she forces veracity at the point of the indiscernible" (p. 411).

The use of genericity for philosophy and culture, outside its original technical environment, is one of the greatest contributions of Badiou's trilogy. In fact, generic processes (love, art, science, politics), offer a complete passage of the categories of Being (multiple, void, nature, infinity) and Event (ultra-one, intervention, fidelity).

On the other hand, one can also find three fundamental concepts in Grothendieck's toposes: $(1 \mathrm{G})$ at the base, the Grothendieck topology $J$ in an adequate category $C$ and its associated site $(C, J) ;(2 \mathrm{G})$ at the approximation level, a collection of sheaves with its gluing properties; $(3 \mathrm{G})$ at the ideal level (or point at infinity), the emerging complete structure of the topos with its limits, exponentials, and classifier object. Many passages of Logics of Worlds are related to situations $(1 \mathrm{G})-(3 \mathrm{G})^{7}$ :

7 The citation numbers refer to pages in the English translation: Alain Badiou, Logics of Worlds. 
$(1 \mathrm{G})$

"What we are attempting here is a calculated phenomenology. [...] Hence a style of formalization that is both more geometrical and more calculating, at the boundary between a topology of localizations and an algebra of forms of order" (pp. 38-39). "The axiomatic deployment of what a place (or a power of localization) is consists in finding the principles of interiority" (p. 411).

"a site is a multiple which happens to behave in the world in the same way with regard to itself as it does with regard to its elements, so that it is the ontological support of its own appearance” (p. 363).

"Logic of the site" and the four forms of change (pp. 369-380).

(2G)

"it is possible to take even further the thinking of the logico-ontological, of the chiasmus between the mathematics of being and the logic of appearing. But one then needs to equip oneself with a more topological intuition and to treat the degrees of the transcendental as operators of the localization of multiple-beings. [...] To use the technical language of contemporary mathematics, this correlation is a sheaf" (p. 197).

"the logical identity of appearing is elucidated particularly well by the theory of complete Heyting algebras” (p. 389).

"The subjectivation of the new body will acquire the creative form of a constant broadening of structural correlations, of the 'visibility' of one structure in another. In particular, 'reading' algebraic structures in topological structures will become the key to contemporary mathematics. With the concept of sheaf, which synthesizes this type of correlation and serves as its general organ, there undoubtedly begins the history of a new body, for which Grothendieck arguably played around 1950 the same role that Galois played around 1830” (p. 475). “(...) let's hold on to the notion, which we have seen at work in both mathematics and poetry, that the sequence world-points-site-body-efficacious part-organ is indeed the generic form of what makes it possible for there to be such things as truths. This authorizes the materialist dialectic to contend that beyond bodies and languages, there is the real life of some subjects” (p. 475).

"the idea which has received the name of 'Grothendieck topos' and the related idea of a sheaf shine in the sky of pure thought” (p. 540). 
"The mathematics of appearing consists in detecting, beneath the qualitative disorder of worlds, the logic that holds together the differences of existence and intensity” (p. 39).

"The infinite of worlds is what saves us from every finite disgrace. [...] We overcome all this when we seize hold of the discontinuous variety of worlds and the interlacing of objects under the constantly variable regimes of their appearances” (p. 514).

Here, the use of a materialist dialectics provides a crossing of the diverse logics of the world, where a "universality of truths" emerges beyond individuals and communities (pp. 5-6).

Finally, three other main ideas govern the behaviour of large cardinals: (1L) the back-and-forth between absoluteness and localisations; (2L) the back-andforth between genericity (ultrapower) and existence (power); (3L) the back-andforth between "A-subjectivity" (the index of absoluteness) and subjectivity (art, science, love, politics: “works of Truth”). Many passages of The Immanence of Truths are now related to situations (1L)- $(3 \mathrm{~L})^{8}$ :

(1L)

“Absolute place V” (p. 61).

negative access to the inaccessible (p. 303).

going beyond the generic (p. 275).

elementary embedding (p. 296).

“point at infinity” (p. 109).

genericity = ultrapower (p. 423).

“transgressions” (p. 307).

“maximalizations" (p. 339).

“partitions” (p. 318).

filtering (p. 335).

8 The citation numbers (for both quotes and glosses) refer to pages in Alain Badiou, L'Immanence des vérités. 
(3L)

A-subjective $=$ index of absoluteness (p. 514).

art: power of form (p. 544).

"science: the power of the letter" (p. 579).

"love: the stage of the Two" (p. 611).

politics: the stage of the Others (p. 639).

Here, by the multiplication and ultralimitation procedures of large cardinals, we can see how the singular, set-theoretic, category of Truth present in Being and Event, is extended to the plural, but again set-theoretic, categories of Truth present in The Immanence of Truths. The capital, singular, letter in the term "Immanence", pointing to the Absolute (the global contradiction $0=1$ ), enters in counterpoint with the lower case, plural, letter in "vérités" (the local realisations at each large cardinal layer). The dialectics of the One and the Many, the contradiction and the models, the forms of incompleteness and completeness, are very nicely explored by Badiou, in order to glue the complexity of mathematics with the complexity of Life and the World. In fact, one can sense that, beyond the set-theoretic intrinsic and natural flavour of large cardinals, a sheaf-theoretic reading is also implicit, first, along cuts and gluings, following increasingly high consistency results (from inaccessibles to superhuges, through Jónsson, Rowbottom, Ramsey, Woodin, Vopenka, "from the top to the bottom (...) something descriptive and baroque” (p. 281), and second, along the fibres of a universal sheaf, where each large cardinal may be seen as a localisation of the Absolute, and each family of large cardinals can be understood through an ultrapower of the Absolute.

\section{An elementary Peircean and category-theoretic reading of the trilogy}

When faced with contemporary thought, we cannot escape a certain transitory ontology $y^{9}$ that at first, terminologically speaking, seems self-contradictory. Nevertheless, although the Greek ontotetês sends us, through Latin translations, to a supposedly atemporal "entity" or to an "essence" that ontology would study, there is no reason, besides tradition, to believe that those entities or essences should be absolute (in a set-theoretic sense, as in Being and Event) and

9 Alain Badiou, Briefings on Existence. A Short Treatise on Transitory Ontology, trans. Norman Madarasz, SUNY, Albany 2006. 
not asymptotic, governed by partial gluings in a correlative evolution between the World and knowledge (in a category-theoretic sense, as in Logiques des mondes). Going beyond the dichotomy, bimodality, in the sense of Jean Petitot ${ }^{10}$, that is, a dynamic (topological) movement in both physical and morphological-structural space, is related to such a state of things, where "things" have to be replaced in fact by "processes". Both prefixes (TRANS-, BI-) offer a suitable ground to understand the wanderings of contemporary thought.

Peirce had already imagined (or discovered, according to our variable ontological commitment) a wonderful phenomenological tool (Peirce 1886) that helps to unravel the multilayered geometry of the (TRANS-, BI-) situation ${ }^{11}$. Phaneroscopy, or the study of the phaneron, i.e. the complete collective spectrum present to the mind, includes the doctrine of Peirce's cenopythagorean categories (with "ceno-" coming from the Greek kaino, "fresh"), which observe the universal modes (or "tints") occurring in phenomena. Peirce's three categories are vague, general, and indeterminate, and can be found simultaneously in every phenomenon. They are interlaced in several levels, but can be prescised (distinguished, separated, detached) following recursive layers of interpretations, in progressively increasingly determined contexts. A dialectics between the One and the Many, the universal and the particular, the continuous and the discrete, the general and the concrete, is multilayered along a dense variety of theoretical and experimental fibres. ${ }^{12}$

Peirce's Firstness detects the immediate, the spontaneous, whatever is independent of any conception or reference to something else. Secondness is the category of facts, mutual opposition, existence, actuality, material fight, action and reaction in a given world. Thirdness proposes a mediation beyond clashes, a third place where the "one" and the "other" enter into a dialogue, the category of sense, representation, synthesis. As Peirce reckons:

By the Third, I understand the medium which has its being or peculiarity in connecting the more absolute first and second. The end is second, the means third.

10 Jean Petitot, Per un nuovo illuminismo, Bompiani, Milano 2009.

${ }_{11}$ Charles Sanders Peirce, “One, Two, Three: An Evolutionist Speculation”, in Writings, Indiana University Press, Vol. 5, pp. 300-301.

${ }_{12}$ For a mathematical introduction to Peirce's architecture, see Fernando Zalamea, Peirce's Logic of Continuity, Docent Press, Boston 2012. 
A fork in the road is third, it supposes three ways. (...) The first and second are hard, absolute, and discrete, like yes and no; the perfect third is plastic, relative, and continuous. Every process, and whatever is continuous, involves thirdness. (...) Action is second, but conduct third. Law as an active force is second, but order and legislation third. Sympathy, flesh and blood, that by which I feel my neighbor's feelings, contains thirdness. Every kind of sign, representative, or deputy, everything which for any purpose stands instead of something else, whatever is helpful, or mediates between a man and his wish, is a Third. ${ }^{13}$

Peirce's vague categories can be “tinctured” with key-words: (1) Firstness: immediacy, first impression, freshness, sensation, unary predicate, monad, chance, possibility; (2) Secondness: action-reaction, effect, resistance, binary relation, dyad, fact, actuality; (3) Thirdness: mediation, order, law, continuity, knowledge, ternary relation, triad, generality, necessity. The three Peircean categories interweave recursively and produce a nested hierarchy of interpretative modulations. Dynamic cognition yields progressive precision through progressive prescision. Both surgery and gluing form part of a ubiquitous topology of comprehension. Intelligence grows with the definition of ever more contexts of interpretation, and the association of finer and finer cenopythagorean tinctures inside each context.

On the other hand, Category Theory offers a multitude of tools to understand the (TRANS-, BI-) imperative. The mathematical theory of categories axiomatises areas of mathematical practice, in accordance with the structural similarities of the objects in question and with the modes of transmission of information between these objects (here, categories are close to methodological and philosophical approximations, sensitive to problems of transference, as in the case of Peircean pragmaticism). As opposed to set theory, where objects are analysed internally as aggregates of elements, the mathematical theory of categories studies objects by way of their external synthetic behaviour, due to the relations of the object with its environment. The objects are like black boxes, which cannot be analysed and broken into smaller interior sub-boxes, and which can be understood only by way of their actions and reactions with the surrounding medium. The modes of knowledge are then essentially relational: the ways in

13 Peirce, “One, Two, Three: An Evolutionist Speculation”, p. 300. 
which the information transmitters behave in the environment constitute the mathematical weaving in which thought grows.

A morphism is universal with respect to a given property if its behaviour with respect to similar morphisms in the category possesses certain uniquely identifying characteristics which distinguish it within the categorical framework. The basic notions of Category Theory related to universality - those of free object and adjointness - respond to deep problems related to the search for relative archetypes and relative dialectics. In fact, after Gödel, the turn in mathematics toward problems of relative consistency (thus, overcoming chimerical longings for absolute foundations) resulted in an explosion of diversity and differentiation in axiomatic mathematical theories, beyond a certain threshold of complexity. Within the resulting multiplicity, in the broad, variable spectrum of the areas of mathematics, Category Theory managed to find some patterns of universality which facilitated processes of local unfolding and also the transcendence of concrete particulars. For instance, in a category, a free object is able to project itself into any object whatsoever taken from a sufficiently wide subclass of the category: it is thus a sort of primordial sign, embodied in all related contexts of interpretation. Hence, relative universals arise beyond relative localisations; these have given a new technical impetus to the classical notions of universality. Although it is no longer possible to presume that we are in a supposed absolute, nor to believe in uniform, stable concepts regarding space and time, Category Theory has reshaped the notion of universality, making it suitable for a series of relative transferences of the universal/free/generic, in which transition is allowed, and in which at the same time it is possible to find remarkable invariants beyond it. ${ }^{14}$

Thus, Category Theory explores the structure of certain generals in a way similar to that of Peirce's late scholastic realism. Indeed, categorical thinking contemplates a dialectics between universal definitions in abstract categories (generic morphisms) and realisations of those universal definitions in concrete categories (structured set classes); moreover, within abstract categories, there may perfectly well be morphisms that are real universals, while at the same

${ }_{14}$ For elaborations of these remarks, see Fernando Zalamea, "A Category-Theoretic Reading of Peirce's System: Pragmaticism, Continuity, and the Existential Graphs”, in New Essays on Peirce's Mathematical Philosophy, ed. Matthew Moore, Open Court, Chicago 2010, pp. 203-233. 
time, not being existent (that is to say, they are not embodied in concrete categories: think, for example, about an initial object, readily definable in abstract categories, but which is not realised in the category of infinite sets, in which initial objects do not exist). In the range of pure possibilities, the pragmatistic maxim has to deal with the idea of universal concepts, logically correct, but which could possibly not turn out to be embodied in bounded contexts of existence (such as, in the case of the three Peircean categories: real universals that may not always adequately be realised in concrete existents within the bounded contexts). The mathematical theory of categories illuminates this kind of situation with a high degree of precision. Category Theory has actually managed to effect the technical construction of a variety of entities, seemingly as elusive as those real universals with no existence, thanks to a very interesting dialectical process between the domains of actual mathematical practice (computational, algebraic, or differential structures, for example) and the possibility of abstract, universal definitions, still not realised in that practice. For instance, following current tendencies in universal algebra and Abstract Model theory [Category Theory has been able to define truly general notions of logic and of relative truth universals, as suitable invariants of given classes of logics.

Using Peirce's Triadicity and Category Theory, Badiou's trilogy Being and Event (BB), Logics of Worlds (LW), and The Immanence of Truths (IT), acquire a simple geometrical structure (see Figure 1): $\mathbf{1}$ (BB)

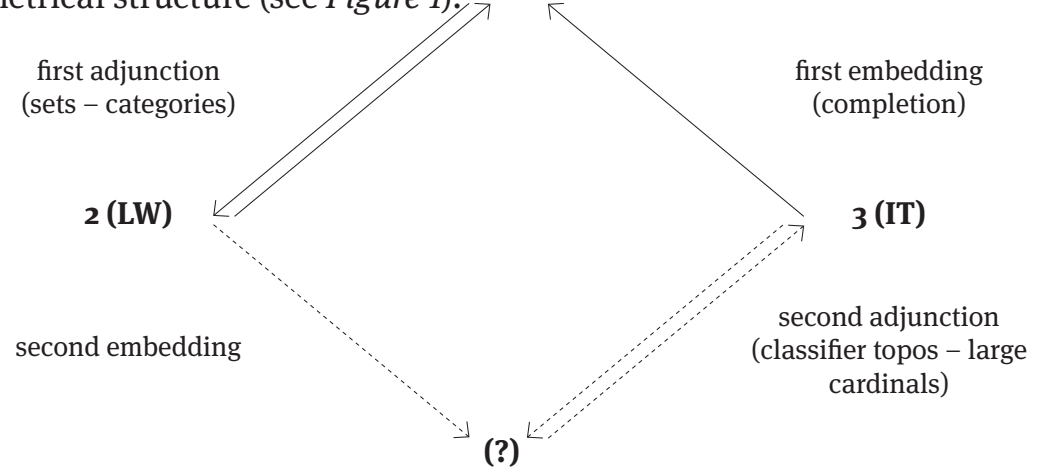


As a (Peircean) First, (BB) proposes a monadic, fresh, intuitive entrance to infinity and the Absolute. As a Second, (LW) acts and reacts against the first naive approximation, and multiplies the range of the World. As a Third, (IT) mediates between the Absolute (inconsistency $\mathrm{o}=1$ ) and the Relative (large cardinals consistency layers). Beyond that, since (IT) can be seen as a completion of (BB), one would expect that a fourth shutter of an extended tetralogy would act as a completion for (LW). The dialectical forces at stake now become pretty clear: on one hand, both sets and categories become fundamental for a thorough understanding of the polarities One-Many, Absolute-Relative, and both of their projections on culture ("works of Truth") enrich the transits of reason and heart; on the other hand, the search for a multilayered geometry of knowledge is embodied in triadic ramifications, iterations, embeddings, projections, and adjunctions.

\section{Some open problems}

Badiou's work is full of questions and suggestions; perhaps what one may appreciate the most in a philosophical reflection. The density of the concepts studied, and the corresponding richness of the perspectives, allow many wanderings. Below we summarise five of those queries:

(i) The study of the pendulum sets - categories, along many of the perspectives presented in the Prague Conference (2018): philosophical openness (Alunni), dualities and dialectics (Guitart), universe $L$ (Berankova), inconsistent multiplicities (Tho), multiverses (Hussey), bordering metaontology (Baki), historical relativity (Barbin), locality and genericity (Cartier), combinatorial time flow (Sumic), Riemann multiplicities (Rabouin), subtraction gestures (Hauser), the logic of True Life (Nesbitt), textual mathematical operations (Bolz), via negativa (Feltham), configuration excesses (Madarasz), etc.

(ii) The construction of a general mediation between Set Theory (analytics) and Category Theory (synthetics), along a third foundational perspective ("horotics"), with axioms for a theory of frontiers and limits, capturing what is common to neighbourhood coverings (analytics) and Grothendieck topologies (synthetics).

(iii) The category-theoretic completion of Logics of Worlds, in correlation with the set-theoretic completion of Being and Event provided by The Immanence of Truths, and the study of the many geometrical features of the situation (Figure 1 above). 
(iv) A comparison of Badiou's Absolute in The Immanence of Truths, with Florensky's Antinomy as the true foundation of mathematics ${ }^{15}$ : the back-and-forth between contradiction and consistency provides a complex and dense multilayered understanding of mathematics, and its projections onto the World.

(v) A comparison of Badiou's poetical understanding of the infinite with Borges's many renderings in El Aleph (1949): transcendence, resistance to any division, the power of internal determinations, the intimate relation to the Absolute. ${ }^{16}$

The development of some of these threads would enhance our understanding of mathematics, the philosophy of mathematics, general philosophy, and cultural studies at large. Our community should be sincerely grateful to Alain Badiou for opening up such possibilities.

\section{References}

Badiou, Alain, L'Être et l'événement, Seuil, Paris 1988

- Court traité d'ontologie transitoire, Seuil, Paris 1998

- Logiques de mondes, Seuil, Paris 2006

- L'Immanence des vérités, Seuil, Paris 2018

Caramello, Olivia, Theories, Sites, Toposes, Oxford University Press, Oxford 2018

Florensky, Pavel, The Pillard and Ground of the Truth, Princeton University Press, Princeton 2004

HoTT, Various Authors, Homotopy Type Theory, Univalent Foundations Project, Princeton 2018

Peirce, Charles Sanders, “One, Two, Three: an evolutionist speculation [1886]”, in Writings: A Chronological Edition, vol. 5., 300-301, Indiana University Press, Bloomington 1993

Petitot, Jean, Per un nuovo illuminismo, Bompiani, Milano 2009

Zalamea, Fernando, “A Category-Theoretic Reading of Peirce’s System: Pragmaticism, Continuity, and the Existential Graphs”, in New Essays on Peirce's Mathematical Philosophy, ed. Matthew Moore, 203-233, Open Court, Chicago 2010

- Peirce's Logic of Continuity, Docent Press, Boston 2012

15 Pavel Florensky, The Pillard and Ground of the Truth, Princeton University Press, Princeton 2004 .

16 Badiou, L'Immanence des vérités, pp. 282-284. 